\title{
Person-Situation Debate Revisited: Phase Transitions with Quenched and Annealed Disorders
}

\author{
Arkadiusz Jędrzejewski (1) and Katarzyna Sznajd-Weron * \\ Department of Theoretical Physics, Faculty of Fundamental Problems of Technology, Wrocław University of \\ Science and Technology, Wrocław 50370, Poland; arkadiusz.jedrzejewski@pwr.edu.pl \\ * Correspondence: katarzyna.weron@pwr.edu.pl; Tel.: +48-71-320-2159
}

Received: 30 June 2017; Accepted: 9 August 2017; Published: 13 August 2017

\begin{abstract}
We study the $q$-voter model driven by stochastic noise arising from one out of two types of nonconformity: anticonformity or independence. We compare two approaches that were inspired by the famous psychological controversy known as the person-situation debate. We relate the person approach with the quenched disorder and the situation approach with the annealed disorder, and investigate how these two approaches influence order-disorder phase transitions observed in the $q$-voter model with noise. We show that under a quenched disorder, differences between models with independence and anticonformity are weaker and only quantitative. In contrast, annealing has a much more profound impact on the system and leads to qualitative differences between models on a macroscopic level. Furthermore, only under an annealed disorder may the discontinuous phase transitions appear. It seems that freezing the agents' behavior at the beginning of simulation-introducing quenched disorder-supports second-order phase transitions, whereas allowing agents to reverse their attitude in time-incorporating annealed disorder-supports discontinuous ones. We show that anticonformity is insensitive to the type of disorder, and in all cases it gives the same result. We precede our study with a short insight from statistical physics into annealed vs. quenched disorder and a brief review of these two approaches in models of opinion dynamics.
\end{abstract}

Keywords: phase transitions; disorder; nonlinear voter model; opinion dynamics

\section{Introduction}

What is more influential in determining a person's behavior-personality or situation? This question is the essence of the person-situation debate, one of the most significant controversies in the history of personality psychology [1]. In the first third of the 20th century, it was possible to believe that social behavior was best understood in terms of personality traits [2]. Thus, the best way to predict or explain a person's behavior in a particular situation would be to examine a personality trait corresponding to the behavior. However, as claimed by Richard E. Nisbett (one of the most eminent social psychologists in the world over the past 40 years), the trait conception as the determinant of social behavior is incorrect. The empirical evidence indicates that only very weak predictability is possible using traditional trait construct [2]. Nevertheless, the person-situation debate is still alive among some personality psychologists [1]. As physicists, we have no intention of resolving this controversy. However, the question of which is a better predictor of an individual's behavior-situation or personality-is crucial in the context of agent-based social simulations. Therefore, the debate inspired us 3 years ago to compare these two approaches (person vs. situation) within a relatively simple yet very interesting model of opinion dynamics—namely, the $q$-voter model $[3,4]$.

As a prime example of an opinion formation model, the $q$-voter dynamics has gained a great deal of attention in recent studies [5-11]. As a spreading mechanism, it finds many applications not only 
in the field of opinion dynamics, but also in modeling the diffusion of innovations, decision-making processes, or expansion of information [11-15]. It was developed by Castellano et al. [3] as a nonlinear version of the voter model [16] which, by contrast, was originally intended to imitate interspecific competitions. In the $q$-voter dynamics, interactions resulting from conformity give rise to interesting ordering phenomena.

However, it is well known that conformity-understood as a change in behavior or belief to accord with others-is not the only type of social response [17]. In social psychology, models of social response concern the identification and delineation of possible responses to social pressure. The basic goal of researchers in this field is to identify the minimum number of variables that is needed to adequately distinguish between responses such as conformity (positive influence) and two types of nonconformity: independence (no influence) and anticonformity (negative influence) [18]. Therefore, later, the $q$-voter model was altered by incorporating two types of stochastic noise which can be thought of as different manifestations of nonconformity: independence and anticonformity [5]. Both kinds of nonconformity tend to destroy the ferromagnetic order. Therefore, the modified model exhibits order-disorder phase transitions. Interestingly, it has occurred that the $q$-voter model with independence exhibits tricriticality; i.e., the switch from continuous to discontinuous phase transition for a critical value of $q=5$. On the other hand, the model with anticonformity displays only continuous phase transition for arbitrary value of $q[5,6]$.

Originally, nonconformity was introduced to the $q$-voter model as a stochastic disturbance, which means that with a certain probability, each agent could behave as a nonconformist or a conformist, which corresponds to the situation-oriented approach. The person-situation debate inspired us to investigate how results would change if we used a person-oriented approach (i.e., where some agents are permanently conformists and other nonconformists). In our previous paper, we have focused on the $q$-voter model with independence with a fixed value of parameter describing the size of the group of influence $q=4$ [4]. At that time, our aim was to examine what the role of the so-called flexibility factor $f$ would be, which describes the probability of change in case of independence. We have shown that this factor influences results only in case of a situation-oriented approach, and for the person-oriented approach, results do not depend on $f$.

Recently, we have realized that we could overlook many interesting results by focusing only on the $q$-voter model with independence and a fixed value of $q=4$. Therefore, we have decided to investigate both versions of the model in this work: model A (conformity + anticonformity) and model I (conformity + independence) for an arbitrary value of $q$. This study allows us to address two previously asked questions at the same time, namely:

1. Do differences between two types of nonconformity that are recognized by social psychologists on the individual (microscopic) level manifest on the society (macroscopic) level? This question has already been addressed in two papers, but only within the situation-oriented approach $[5,6]$.

2. Do differences between two types of approach (situation- and person-oriented) introduced on the individual (microscopic) level manifest on the society (macroscopic) level? This question has already been addressed in [4], but only within the $q$-voter model with independence with $q=4$.

From a physical point of view, the second question corresponds to the problem of annealed (situation) vs. quenched (person) disorder. Therefore, in the next section we will briefly describe some insights from statistical physics into this issue. Then, in Section 3 , we will briefly review what kinds of approaches are used in opinion dynamics models. In Section 4 we will describe the model that will be studied in this paper, and in Section 5 we will present analytical results for our model. We will conclude in the last section.

\section{Quenched and Annealed Disorder}

Disorder is an inherent part of any real system. It introduces inhomogeneities into the models imitating external fluctuations, structural changes, or impurities which are present in the real world. 
Developing a fundamental understanding of disorders is, therefore, one of the essential aspects in modern statistical physics. They play especially important role in modeling complex systems by appearing in the network structures, for instance, changing the link arrangements or node degrees [19-21]. Disorders may also modify the character or the strength of interactions [22,23]. Regardless of their origin and nature, we can distinguish two types of disturbances based on the time-scale of their appearance [23-25]. Disorder is said to be quenched when it does not evolve in time. It is permanently frozen through the entire period of observation, so the disorder fluctuation time-scale is considerably longer than the rate of changes of dynamical variables which describe the system. In such a case, the impurities are not allowed to move, the network structure is fixed, and the interactions are established. Therefore, in order to study the thermodynamic properties of the model, the partition function is determined for the specific disorder realization, and only the state functions are averaged over distinct distributions of disturbances. A completely different approach should be applied when both time-scales are comparable. Then, the randomness connected with the disorder is not fixed, and it vacillates in time. If this is the case, we talk about the annealed disorder, and now, the distribution of disturbances has to be taken into account already in the calculation of the partition function.

In general, these two specific time regimes give completely different results. The annealed disorder connected with the network structure introduces additional fluctuations into the system which may lower the transition temperature as in $[23,26]$, or even cause so-called robust critical behavior [26]. Furthermore, when we consider scale-free networks with power law tails in the degree distribution, the annealed system exhibits anomalous finite-size scaling in contrast to the quenched one [19]. On the other hand, the same type of disorder but this time associated with the character and strength of interactions may increase the critical temperature [27] so that the final system is more stable. Recently, it has been shown that some dynamics are more sensitive to the changes of disturbance kind. This effect is especially pronounced in the Ising model with Metropolis dynamics, where the graph annealing can even switch the type of phase transition from continuous to discontinuous [28].

\section{Person vs. Situation Approach in Models of Binary Opinion Dynamics}

Richard E. Nisbett claims that behavior can be predicted much better from the social setting than from personality traits. Indeed, in the strong version of his position, personality is only an illusion-albeit a quite powerful one [2]. On one hand, he calls the person-oriented approach, after Lewin, a naive psychology. On the other, he explains why the idea of personal traits as a dominant predictor of behavior is so attractive and natural. He compares naive psychology to ancient physics, when the behavior of objects was understood exclusively in terms of object itself: A stone sinks when placed in water because it has property of "gravity"; a piece of wood floats because it has the property of "levity" [2]. In fact, this example is not very convincing for a physicist, and following this argument, we could argue that probably both personality and situation are important. It is true that the gravitational and buoyant forces act on both items, but stone and wood behave differently because of an important property of any material, namely density (i.e., its "personal trait"). Anyway, as social psychologists claim, the empirical evidence indicates that personality is a very weak predictor of an individual's behavior, and the situation can completely dominate over the personal traits $[2,17]$.

The question of which approach is more fundamental and better describes real systems is particularly important for social agent-based models, such as models of opinion dynamics. Frequently, these models originate from statistical physics. For instance, the Ising model with ferromagnetic interactions in an external magnetic field were used to interpret collective social phenomena and decision-making processes $[29,30]$. As in the $q$-voter model, an opinion was mapped into a two-state variable, but the interactions were given by the function measuring the degree of convergence in the society which brings to mind the Hamiltonian function. Instead, in the $q$-voter model, the interactions are defined by the microscopic dynamical rules described in the next section. Moreover, in the proposed 
model, the individual bias connected with personal believes and experiences was introduced by a quenched local field, and the annealed approach was not considered.

Nowadays, in sociophysics, we can find models of opinion dynamics which incorporate both approaches. For example, Galam has introduced a contrarian behavior to his model in the spirit of annealed disorder [31]. This means that in each time step, a fixed fraction $a$ of the agents is randomly chosen to be contrarians (i.e., an individual that is tossed as a contrarian in one time step may act as a non-contrarian in the next time step) [32]. Afterwards, different modifications have been proposed to Galam's model, including a variable in time fraction of contrarians or one-sided contrarians, the antagonistic behavior of which is restricted only to the global majority opinion, but all of them showed up in the annealed form [33]. Stauffer and Martins introduced another-in their opinion, more realistic - quenched approach, and showed that results for these two approaches are different for the original Galam's model [32]. In their approach, a fraction $a$ of the population is randomly selected as contrarians at the beginning of the simulation, and they keep this trait until the end of the simulation. As the order parameter they have chosen (as usual in this kind of model) magnetization; i.e., the number of individuals with positive opinion minus that of negative opinion, divided by the total size of the population. It has been shown that continuous order-disorder phase transition is observed within both approaches, but the critical fraction of contrarians is much higher for the quenched version.

In a similar spirit, Schneider has introduced three different types of behavior in addition to conformity to the Sznajd model [34]: unconvincibles (people who cannot be convinced at all), opportunists (agents who adopt the opinion of the majority of all others, instead of neighbors), and contrarians (in his approach, these agents cannot be convinced by their neighborhood, but they always adopt the opposite opinion of the majority of all other people). He has investigated these three types of behavior within quenched or annealed approaches. Similarly, as for Galam's model, only quantitative differences between these two approaches have been seen. In general, differences have been observed in the exit time (i.e., time needed to reach an absorbing state) - evolution was faster for the annealed approach. One should notice that the contrarian behavior investigated by Schneider is of a mean-field type, contrary to Galam's idea, who assumed that contrarians take the opposite opinion to the neighborhood and not to all other people. The importance of the influence range in opinion dynamics was later studied by Javarone in both quenched and annealed approaches [35]. Based on the majority rule, he introduced conformists and contrarians to his model. In one scenario, the interactions have only local dimension (i.e., the nonconformist behavior is restricted only to the social circle of each agent), whereas in the second scenario this behavior is related to the whole population. Once again, the quantitative differences between two disorders were observed-the annealed model turns out to exhibit higher values of the magnetization.

Just as Stauffer and Martins argued that the quenched approach is more realistic, Lama et al. claimed that introducing a given fraction of contrarians in the initial state was artificial. Therefore, Lama et al. introduced the probability of being contrarians, as a parameter, to the Sznajd model [36]. This means that with certain probability each agent can behave like a contrarian, and with complementary probability, it behaves like a conformist, which corresponds to the annealed approach.

Galam and Jacobs have noticed that in addition to contrarian behavior, another type of behavior is also quite current in real opinion dynamics; namely, inflexible attitude. They have also assumed that the inflexible attitude is a quenched individual state: inflexible agents keep their opinion always unchanged [37]. Inflexibles and unconvincibles are in fact the same type of behavior, which corresponds to independence. Another type of independent agent has been introduced within the voter model by Mobilia under the name "zealot" [38]. Originally, the zealot is an individual who favors one opinion, and thus, it is allowed to change its state without regard to its neighbors. Recently, the idea of inflexible zealots, which never change their opinion, has also been investigated within the $q$-voter model $[7,8]$. Zealots have been introduced within the quenched approach, contrary to independence which has been introduced to the $q$-voter model as an annealed disorder $[5,6]$. Unfortunately, zealotry behavior 
cannot be compared directly with independence because zealots in the $q$-voter model are inflexible, whereas under independence an agent can still change its opinion but independently of others.

Applications of opinion dynamics also range over the evolutionary game theory. Lately, the significance of conformists has been analyzed in the spatial public goods game, but only in the spirit of quenched disorder so that individuals could not change their attitude [39]. It turns out that social influence may cause different system behavior, leading to full cooperation or bistable equilibria.

The above brief review shows that indeed different approaches are used, and for some physicists the annealed approach seems to be more realistic, whereas for others quenched disorder is better, which recalls the person-situation debate among psychologists. However, it seems that reality is more complex, and personal long-term traits interact with the immediate situation to influence individuals' behavior. As noted by Goldstein: even the most violent individual is not perpetually violent [40]. It has also been shown empirically that situational variables are more important in predicting immediate behavior in specific situations, whereas personal traits describe the long-term average behavior [41]. Taking these findings into account, one could speculate that in describing opinions related to important fundamental issues such as religion one should use the quenched approach. Probably for the same reason, the quenched approach would be more suitable to describe political opinions. On the other hand, in modeling different marketing phenomena like diffusion of innovation, the annealed approach would be more realistic. Apart from which approach is more realistic, physicists are interested in a comparison between quenched and annealed approaches [4,32,34]. In all described examples, the differences between approaches were only quantitative: visible in the shift of the critical value or in the exit time. Here, we will show that a systematic study of the $q$-voter model with two types of nonconformity for arbitrary $q$ reveals more profound differences.

\section{Model Description}

Within the $q$-voter model, we consider $N$ agents placed on a complete graph, so that each node of a network is associated with only one autonomous voter characterized by a binary variable $s_{i}= \pm 1$, $i \in\{1, \ldots, N\}$. The chosen topology implies that all individuals are neighbors and can interact mutually. Although such structures may be too simple and unrealistic to model large social networks, they are decent representations of interactions within small groups [14]. Additionally, they allow for analytical treatment based on the mean-field approach, and are therefore often incorporated into analyses $[3,5,8]$. More complex structures are also investigated as underlying frameworks for the $q$-voter dynamics $[10,42]$, but for simplicity, we consider only a fully connected graph. Interactions in the system are driven by three factors interpreted as different social responses: conformity, independence, and anticonformity [43]. Only the first has ordering properties, whereas the other two tend to disturb the order, and thus they are regarded as stochastic noise. In previous papers [5,6], two distinct models were considered. Both of them include conformity as ordering force, but the role of disorder is once enacted by independence and once by anticonformity. In both approaches, in every elementary time step, we choose at random an agent which is reconsidering its opinion and a group of influence comprised of $q$ of its nearest neighbors. The group is called $q$-panel and tries to exert social pressure on the chosen voter. With probability $1-p$, the agent conforms to this pressure and embraces the same opinion of the $q$-panel, but only if the group is unanimous (i.e., all $q$ individuals are in the same state). Otherwise, the opinion of the chosen voter remains unchanged. With complementary probability $p$, on the other hand, it acts as a nonconformist, and therefore, two different scenarios may happen depending on the specific disordering interactions incorporated in the studied $q$-voter model:

- Model I (Independence + Conformity): the voter autonomously decides whether to change its viewpoint, and it does not rely on its peers' opinions. In the simulation, it is realized by changing the agent's state to the opposite one with probability $1 / 2$. This social response refers to independence [43].

- Model A (Anticonformity + Conformity): the chosen agent disagrees with the group. It does not submit to social norms, and it teasingly adopts the opposite opinion to the $q$-panel's, provided 
that the group of influence is unanimous. If it is not, the chosen agent preserves the viewpoint it had before. This social response refers to anticonformity [43].

It was shown that these two different types of noncomformity-identified at microscopic level-lead to distinctive macroscopic behavior of the system. From the point of view of social science, it means that differences between responses to social pressure recognized by psychologists at the individual level may manifest on a large scale of whole societies [6,43]. However, in most studies and simulations, the agent's behaviors were determined by situational factors and were not connected with personal traits. So, the parameter $p$ was the same for all agents, and the same voter could be either conformist or nonconformist in different time steps. This approach introduces an annealed disorder to the system, since individuals may change their behavior during one simulation and act once as conformists and once as nonconformists. The quenched disorder, on the other hand, corresponds to the situation in which before the simulation we determine whether an agent is conformist or nonconformist, and its attitude does not change in time. In order to achieve that and additionally make both approaches comparable, at the beginning of simulation, we randomly assign the type of behavior to all agents. With probability $p$, a given agent becomes a nonconformist, and with the complementary probability $1-p$, it turns into a conformist. After this assignment, the agent's attitude stays fixed. Later on, the mechanism behind the opinion change is the same as in the annealed model. In every elementary time step, we choose an agent at random, and depending on this assigned attitude, the agent decides whether to change its opinion.

Both described variants of the $q$-voter dynamics-model I with independence and model A with anticonformity-have already been studied in terms of annealed disorder $[5,6]$. In this work, we analyze the quenched approach to these models, which has not been investigated before except for the particular case where $q=4$ and only for model I [4]. Additionally, we recall the previous results in order to compare both quenched and annealed approaches.

To maintain the review character of previous sections and for clarity of presentation, we describe below algorithms for all models considered in this work.

\subsection{Model I (Independence + Conformity) within the Annealed Approach}

Initialization (set time $t=0$ ): for each node of a graph $i=1, \ldots, N$ choose a random number from uniform distribution $r_{i} \sim U(0,1)$. If $r_{i}<c(0)$ then assign to the node a state $S_{i}(0)=1(\uparrow)$; otherwise, $S_{i}(0)=-1(\downarrow)$. This means that initially the concentration of up-spins is equal to $c(0)=N_{\uparrow}(0) / N$, where $N_{\uparrow}(0)$ is a number of up-spins at $t=0$. Usually one of two types of initial state is used: ordered $(c(0)=1)$ or random $(c(0)=1 / 2)$. After initialization, the system evolves according to the following algorithm:

1. Choose randomly and uniformly $i$ from the set $\{1,2, \ldots, N\}$ and go to 2 .

2. Choose a random number from uniform distribution $r \sim U(0,1)$ and go to 3 .

3. If $r<p$ then $S_{i}(t+\Delta t)=-S_{i}(t)$ with probability $1 / 2$, and with the same probability, $S_{i}$ remains unchanged (independence), set $t=t+\Delta t$ and go to 1 ; otherwise, go to 4 .

4. Choose randomly a group of $q$ distinct neighbors of the chosen $i$-th node: $n_{1}, n_{2}, \ldots, n_{q} \in$ $\{1,2, \ldots, N\}$ and go to 5 .

5. If all $q$ neighbors are in the same state, the chosen spin $S_{i}$ takes the same state as its $q$ neighbors (conformity); i.e., $S_{i}(t+\Delta t)=S_{n_{q}}(t)$, set $t=t+\Delta t$ and go to 1 .

As usual, $\Delta t=1 / N$, which means that the time unit consists of $N$ elementary updates.

\subsection{Model A (Anticonformity + Conformity) within the Annealed Approach}

Initialization is the same as in Model I within the annealed approach, described in Section 4.1. After initialization, the system evolves according to the following algorithm:

1. Choose randomly and uniformly $i \in\{1,2, \ldots, N\}$ as well as $q$ distinct neighbors of the $i$-th node: $n_{1}, n_{2}, \ldots, n_{q} \in\{1,2, \ldots, N\}$ and go to 2 . 
2. If all $q$ neighbors are in the same state, they will influence $S_{i}(t)$ and thus go to 3 ; otherwise, set $t=t+\Delta t$ and go to 1 .

3. Choose a random number from uniform distribution $r \sim U(0,1)$ and go to 4 .

4. If $r<p$, then $S_{i}(t+\Delta t)=-S_{n_{q}}(t)$ (anticonformity); otherwise, $S_{i}(t+\Delta t)=S_{n_{q}}(t)$ (conformity), set $t=t+\Delta t$ and go to 1 .

\subsection{Model I (Independence + Conformity) within the Quenched Approach}

Initialization (set time $t=0$ ): for each node of a graph $i=1, \ldots, N$ choose two random numbers $r_{i}, z_{i} \sim U(0,1)$. If $r_{i}<c(0)$, then assign to the node a state $S_{i}(0)=1(\uparrow)$; otherwise, $S_{i}(0)=-1(\downarrow)$. Additionally, set the type of the node, which will be constant in time: if $z_{i}<p$ then spin $S_{i}$ is independent; otherwise, it is a conformist. After initialization, the system evolves according to the following algorithm:

1. Choose randomly and uniformly $i$ from the set $\{1,2, \ldots, N\}$ and go to 2 .

2. If the chosen spin is independent, then $S_{i}(t+\Delta t)=-S_{i}(t)$ with probability $1 / 2$, and with the same probability it remains unchanged; set $t=t+\Delta t$ and go to 1 . Otherwise, go to 3 .

3. Choose randomly a group of $q$ distinct neighbors of the chosen $i$-th node: $n_{1}, n_{2}, \ldots, n_{q} \in$ $\{1,2, \ldots, N\}$ and go to 4 .

4. If all $q$ neighbors are in the same state, the chosen spin $S_{i}$ takes the same state as its $q$ neighbors (i.e., $\left.S_{i}(t+\Delta t)=S_{n_{q}}(t)\right)$, set $t=t+\Delta t$ and go to 1 .

\subsection{Model A (Anticonformity + Conformity) within the Quenched Approach}

Initialization is almost the same as in Model I within the quenched approach, described in Section 4.3. The only difference is that instead of independent spins we have anticonformists. Thus, if $z_{i}<p$ then the spin $S_{i}$ is an anticonformist; otherwise, it is a conformist. After initialization, the system evolves according to the following algorithm:

1. Choose randomly and uniformly $i \in\{1,2, \ldots, N\}$ as well as $q$ distinct neighbors of the $i$-th node: $n_{1}, n_{2}, \ldots, n_{q} \in\{1,2, \ldots, N\}$ and go to 2 .

2. If all $q$ neighbors are in the same state, they will influence $S_{i}(t)$ and thus go to 3 ; otherwise, set $t=t+\Delta t$ and go to 1 .

3. If $S_{i}$ is a conformist then $S_{i}(t+\Delta t)=S_{n_{q}}(t)$; otherwise, $S_{i}(t+\Delta t)=-S_{n_{q}}(t)$. Set $t=t+\Delta t$ and go to 1 .

\section{Results}

In order to quantitatively describe the behavior of the system, we define an up-spin concentration as a fraction of voters with a positive opinion (i.e., $s_{i}=1$ ). If we denote by $N_{\uparrow}$ the total number of agents in the state $s_{i}=1$, then the up-spin concentration has the following form:

$$
c=\frac{N_{\uparrow}}{N},
$$

and it might be interpreted as a probability of finding a voter with a positive opinion in the system.

Since we consider a random sequential updating scheme, in every elementary time step, only a single agent can change its opinion. There are three possible scenarios. The total amount of up-spins $N_{\uparrow}$ may increase by 1 , decrease by 1 , or remain unchanged. Hence, a flip corresponds to a change in the concentration $c$ by $1 / N$, and the following transition rates can be written:

$$
\begin{aligned}
& \gamma^{+}=\mathrm{P}\left(c \rightarrow c+\frac{1}{N}\right), \\
& \gamma^{-}=\mathrm{P}\left(c \rightarrow c-\frac{1}{N}\right) .
\end{aligned}
$$


Therefore, the time evolution of the system is governed by the rate equation

$$
c(t+\Delta t)=c(t)+\frac{1}{N}\left(\gamma^{+}-\gamma^{-}\right) .
$$

Based on the above formalism, the annealed version of the $q$-voter model with independence and anticonformity was studied in $[5,6]$. Herein, we adopt the same methodology in order to describe the model with quenched agent attitudes, which reflects the person approach.

At the beginning, we divide our society comprised of $N$ agents into two groups. The first one incorporates exclusively nonconformists, and its size is $N_{1}=p N$. The second one gathers only conformists and consists of $N_{2}=(1-p) N$ agents. Thanks to the above partition, we still have the same probability $p$ of finding a nonconformist as in the annealed approach, which makes both scenarios comparable. Furthermore, we can write down explicitly chances for a flip in each group, and consequently, find stationary values of up-spin concentrations from the following condition:

$$
\gamma^{+}-\gamma^{-}=0
$$

In order to do so, let us consider separately the $q$-voter model with independence and anticonformity.

\subsection{Model I: Independence + Conformity}

For the group of independent agents:

$$
\begin{aligned}
& \gamma_{1}^{+}=\frac{1}{2}\left(1-c_{1}\right), \\
& \gamma_{1}^{-}=\frac{1}{2} c_{1},
\end{aligned}
$$

where $c_{1}$ refers to the up-spin concentration among independent agents. From Equation (4) (i.e., equalizing above equations), we get the stationary concentration of independent agents with a positive opinion $c_{1}=1 / 2$. For the second group comprised of only conformists, we write down similar formulas:

$$
\begin{aligned}
& \gamma_{2}^{+}=\left(1-c_{2}\right) c^{q}, \\
& \gamma_{2}^{-}=c_{2}(1-c)^{q} .
\end{aligned}
$$

This time, we obtain the stationary concentration of conformists with a positive opinion:

$$
c_{2}=\frac{c^{q}}{c^{q}+(1-c)^{q}} .
$$

Having $c_{1}$ and $c_{2}$, we are able to determine the overall up-spin concentration by using the following formula

$$
c=p c_{1}+(1-p) c_{2} .
$$

Combining Equation (8) with both concentrations, we have the final result

$$
p=2 \frac{c(1-c)^{q}-(1-c) c^{q}}{(1-c)^{q}-c^{q}} .
$$

The corresponding formula to Equation (9) for the annealed approach obtained in [5] has the following form

$$
p=\frac{c(1-c)^{q}-(1-c) c^{q}}{c(1-c)^{q}-(1-c) c^{q}+1 / 2-c} .
$$


In order to better comprehend differences between the above results (i.e., Equations (9) and (10)), we plot phase diagrams which represent the impact of the model parameter $p$ on the stationary values of the up-spin concentration. Figure 1a presents phase diagrams obtained for the annealed $q$-voter model with independence, obtained from Equation (10), whereas Figure $1 \mathrm{~b}$ shows phase diagrams for the quenched model, obtained from Equation (9). The most striking difference is the change of transition type from discontinuous in the annealed (situation) approach, to continuous in the quenched (person) approach, for $q \geq 6$. It seems that freezing the agent behaviors at the beginning of the simulation, introducing quenched disorder, supports second-order phase transitions, whereas allowing agents to reverse their attitude in time, incorporating annealed disorder, supports discontinuous ones. Figure 2a additionally presents the decomposition of the overall up-spin concentration into the concentration of adopted individuals among independent agents $c_{1}$ and among conformists $c_{2}$.
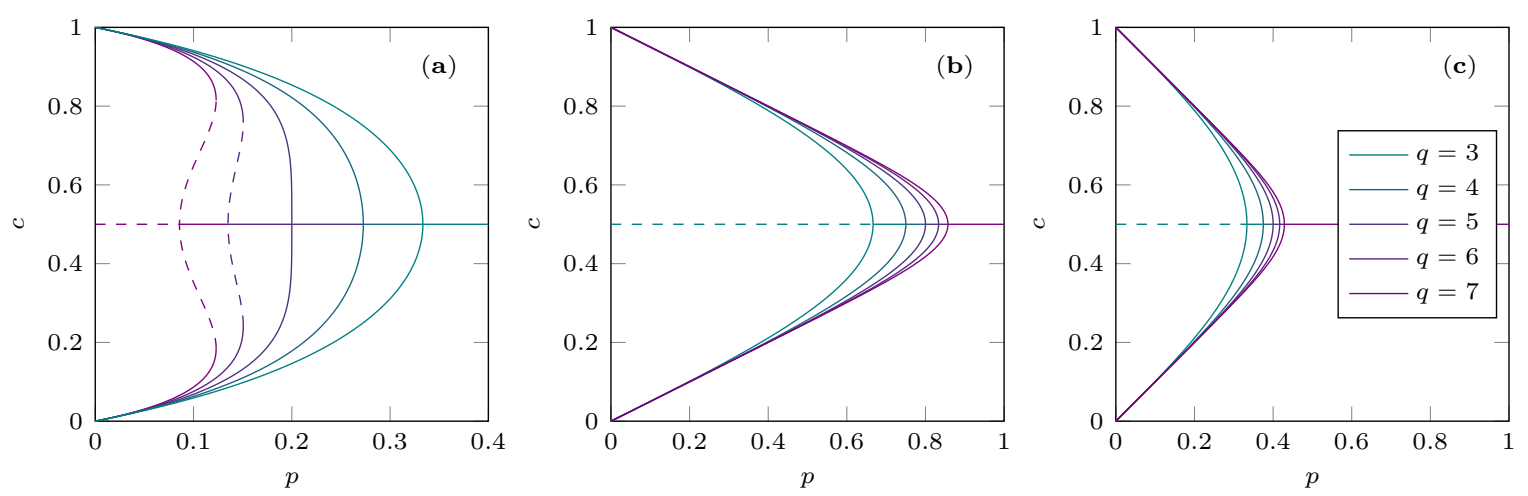

Figure 1. Phase diagrams for the $q$-voter model with independence and anticonformity within annealed and quenched approaches. Darker lines correspond to larger groups of influence (i.e., higher values of q). (a) Annealed model with independence, Equation (10); (b) Quenched model with independence, Equation (9); and (c) Model with anticonformity where annealed and quenched approaches give exactly the same results. Note that for the quenched disorder, we always have continuous phase transitions regardless of the stochastic driving type. Annealed disorder, on the other hand, reveals not only quantitative but also qualitative differences between independence and anticonformity. For independence, transition type changes from continuous $q \leq 5$ to discontinuous $q \geq 6$, whereas for anticonformity all transitions are continuous.
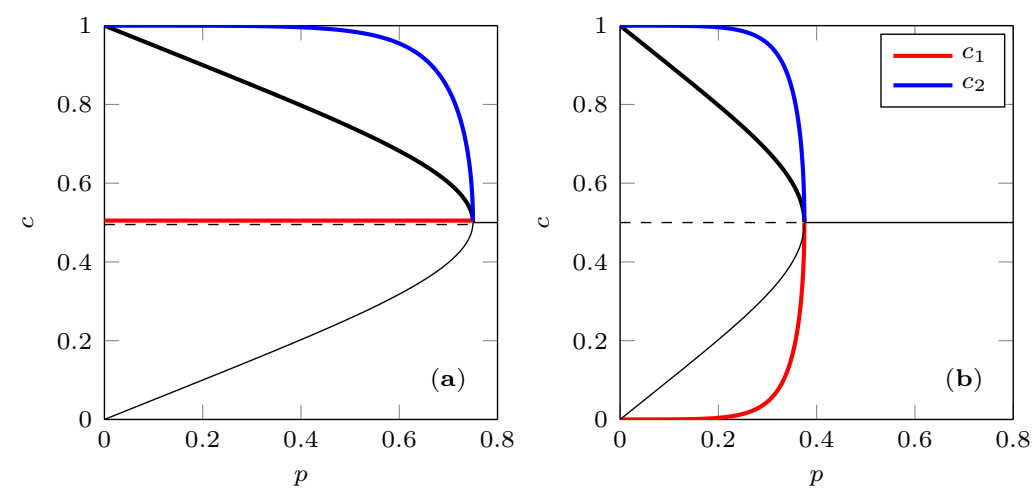

Figure 2. Phase diagrams for the quenched model driven by stochastic noise: (a) Independence and (b) Anticonformity. Black lines correspond to the overall up-spin concentrations. The upper parts of the diagrams-the thick black lines-were decomposed into two colored curves which refer to the up-spin concentrations among specific social groups, so that Equation (8) is fulfilled: blue lines stand for conformists and red ones for (a) independent individuals and (b) anticonformists. The decomposition of the lower parts of the diagrams constitutes a mirror image along $c=1 / 2$. 


\subsection{Model A: Anticonformity + Conformity}

A similar analysis can be done for the $q$-voter model with anticonformity. Once again, we divide our community into two groups. The first one consists of $N_{1}=p N$ agents, and comprises only anticonformists. The second one, as previously, combines conformists in the number of $N_{2}=(1-p) N$. The transition rates have the forms

$$
\begin{aligned}
& \gamma_{1}^{+}=\left(1-c_{1}\right)(1-c)^{q}, \\
& \gamma_{1}^{-}=c_{1} c^{q},
\end{aligned}
$$

and the equations lead to the following stationary value of up-spin concentration

$$
c_{1}=\frac{(1-c)^{q}}{(1-c)^{q}+c^{q}}
$$

In the case of conformity, we have the same formulas derived before Equations (6) and (7). Using Equation (8), we are able to obtain the final result for the stationary value of up-spin concentration

$$
p=\frac{c(1-c)^{q}-(1-c) c^{q}}{(1-c)^{q}-c^{q}}
$$

The associated formula for the same $q$-voter model with anticonformity but within the annealed approach is identical and derived in [5]. Therefore, the $q$-voter model with anticonformity is insensitive to switching between types of disorder, in contrast to the model with independence. The phase diagrams for this quenched disorder are presented in Figure 1c and in Figure 2b; we can see once again the decomposition of the overall up-spin concentration into $c_{1}$ and $c_{2}$. Note that in the case of quenching, only continuous phase transitions are present in the system, regardless of the stochastic driving type. Thus, the differences between models with independence and anticonformity are only quantitative. The model with independence exhibits higher stationary values of up-spin concentration than the model with anticonformity at the same levels of parameter $p$; compare Figure $1 \mathrm{~b}, \mathrm{c}$. It is worth noting that the stationary values of up-spin concentrations are equal for both quenched models with independence and anticonformity under the condition that the level of independence is twice as large as the level of anticonformity; compare Equations (9) and (13). Even so, Figure 3 shows that the trajectories in a phase space are different for these cases. Additionally, these differences are also visible in the corresponding time evolutions presented in Figure 4.
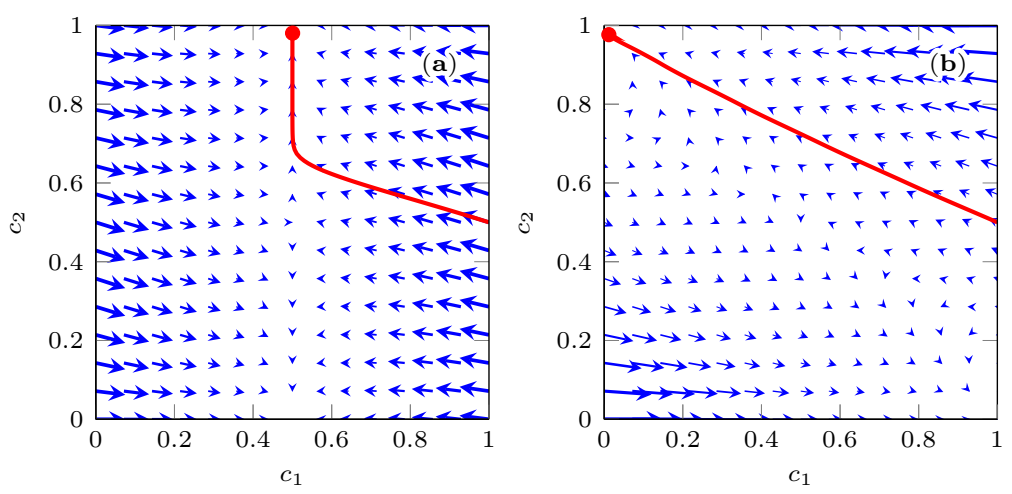

Figure 3. Phase portraits for the $q$-voter model with quenched disorder and the group of influence comprised of $q=4$ agents. The case with (a) independence at level $p=0.5$ and (b) anticonformity at level $p=0.25$. Arrow size indicates the strength of the flow in the phase space. Red curves illustrate trajectories of a system starting from the initial point $\left(c_{1}, c_{2}\right)=(1,0.5)$. Red dots refer to stable points. 

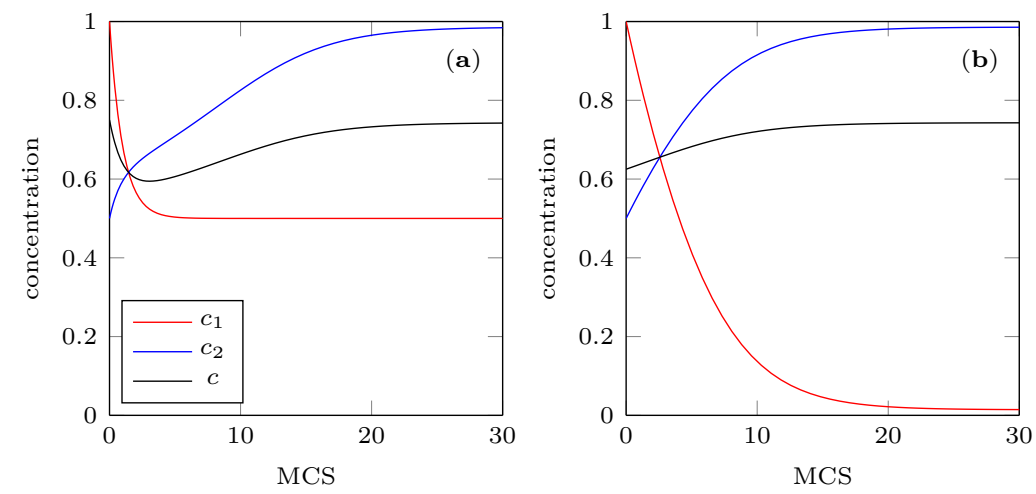

Figure 4. The time evolution, measured in the Monte Carlo steps (MCS), of the concentrations for the quenched system with (a) independence at the level $p=0.5$ and (b) anticonformity at the level $p=0.25$. In both cases, the influence group consists of $q=4$ agents, and the starting point equals $\left(c_{1}, c_{2}\right)=(1,0.5)$. Note that the same parameters are used in Figure 3.

\section{Conclusions}

Understanding the role played by impurities on the nature of phase transitions has been one of the key issues in the theory of phase transitions for years [44,45]. Brout was probably the first to point out that random systems can have two distinct kinds of thermodynamic behavior [46,47]: quenched or annealed. Initially, the effect of quenched bond randomness in the systems, which in its pure version exhibits continuous phase transitions, has been extensively studied and understood within the Harris criterion [48]. The role of quenched disorder in the system with discontinuous phase transitions is less understood. However, it has been argued and then shown in several cases that quenched disorder could induce continuous phase transitions [44]. Exactly this effect has been observed in this paper for the $q$-voter model with independence, in which the quenched disorder changed the character of the phase transitions from discontinuous to continuous.

It is not surprising that investigating how the type of randomness influences the system is interesting for physicists, because this is the issue of great importance from both experimental and theoretical perspectives [44]. However, it maybe be astonishing for physicists (at least, it was for us) that the analogous problem is a key point of the most significant controversy in the history of psychology. Of course, the questions asked by physicists and psychologists are completely different. Let us summarize this difference in a slightly oversimplified way: physicists are interested in the role of a particular type of randomness in systems as a whole (on the macroscopic scale), whereas psychologists ask if a given type of randomness in a system can exist, and they are not interested in how it will affect the system (social group, society). However, as physicists we believe that answering the first question can be helpful in answering the second. In fact, this is what statistical physicists do-in the end, they try to understand how the system works by looking at the macroscopic outcome of the microscopic model and validating its correctness. Such a validation is of course much more difficult in social sciences. Nevertheless, we can still ask a "physical question": How does the system in which only personal traits influence the individual's attitude behave on a macroscopic scale in comparison to the system in which only situation matters, and individuals have no personal trait? This question was asked 3 years ago within the $q$-voter model with independence and $q=4$ [4]. Here, we revise the study, investigating two versions of the model for an arbitrary value of $q$ : the $q$-voter model with independence (Model I) and the $q$-voter model with anticonformity (Model A).

First of all, we have shown that the $q$-voter model with anticonformity is fully robust to the type of randomness. Exactly the same results have been obtained for the annealed and quenched disorder, which is quite surprising. From the psychological perspective, it means that if anticonformity and conformity were the only types of social response, the person-situation debate would be senseless 
from the sociological (macroscopic) perspective. On the other hand, it has been shown that the $q$-voter model with independence is very fragile to the type of randomness. In this case, quenched disorder changes the character of the phase transition from discontinuous to continuous.

The second interesting result is related to the differences between two types of nonconformity. This question was asked for the first time in [5] within the annealed approach (situation approach), and it has been shown that the model with anticonformity behaves qualitatively differently than the model with independence. However, as we have shown here, quenched disorder (person approach) completely kills these differences. A simple rescaling of the level of nonconformity makes these two models identical. In our opinion, this result is particularly interesting from a psychological perspective because it is believed that independent people influence society much more strongly than anticonfomists. Therefore, results obtained here are in line with the claims of social psychologists that situation is more important than personality in predicting an individual's behavior [2,17].

Of course, all of these results are valid only for the complete graph, which allowed for the analytical treatment. It is an interesting task for the future to check how results would change in complex networks.

Acknowledgments: The work was partially supported by funds from the National Science Centre (NCN, Poland) through grants no. 2016/21/B/HS6/01256 (to K.S.W.) and no. 2016/23/N/ST2/00729 (to A.J.). K.S.W. is grateful to Paul Nail, social psychologists, for fruitful discussions and paying attention to the work by Nisbett on personality vs. situation.

Author Contributions: A.J. has done all the calculations, prepared all the figures, wrote Sections 2 and 5. K.S.W. has designed researched and wrote Section 3 . All authors interpreted results and wrote Sections 1,4 and 6 . Both authors read and approved the final manuscript.

Conflicts of Interest: The authors declare no conflict of interest.

\section{References}

1. Donnellan, M.B.; Lucas, R.E.; Fleeson, W. Introduction to personality and assessment at age 40: Reflections on the legacy of the person-situation debate and the future of person-situation integration. J. Res. Pers. 2009, 43, 117-290.

2. Nisbett, R.E. The trait construct in lay and professional psychology. In Retrospectives on Social Psychology; Festinger, L., Ed.; Oxford University Press: Oxford, UK, 1980; pp. 109-130.

3. Castellano, C.; Munoz, M.A.; Pastor-Satorras, R. Nonlinear q-voter model. Phys. Rev. E 2009, 80, 041129.

4. Sznajd-Weron, K.; Szwabiński, J.; Weron, R. Is the person-situation debate important for agent-based modeling and vice-versa? PLoS ONE 2014, 9, e112203.

5. Nyczka, P.; Sznajd-Weron, K.; Cislo, J. Phase transitions in the q-voter model with two types of stochastic driving. Phys. Rev. E 2012, 86, 011105.

6. Nyczka, P.; Sznajd-Weron, K. Anticonformity or independence?-Insights from statistical physics. J. Stat. Phys. 2013, 151, 174-202.

7. Mobilia, M. Nonlinear $q$-voter model with inflexible zealots. Phys. Rev. E 2015, 92, 012803.

8. Mellor, A.; Mobilia, M.; Zia, R.K.P. Heterogeneous out-of-equilibrium nonlinear $q$-voter model with zealotry. Phys. Rev. E 2017, 95, 012104.

9. Siedlecki, P.; Szwabiński, J.; Weron, T. The interplay between conformity and anticonformity and its polarizing effect on society. J. Artif. Soc. Soc. Simulat. 2016, 19, 9.

10. Jedrzejewski, A. Pair approximation for the q-voter model with independence on complex networks. Phys. Rev. E 2017, 95, 012307.

11. Rodriguez, N.; Bollen, J.; Ahn, Y.Y. Collective dynamics of belief evolution under cognitive coherence and social conformity. PLoS ONE 2016, 11, e0165910.

12. Kowalska-Pyzalska, A.; Ćwik, K.; Jedrzejewski, A.; Sznajd-Weron, K. Linking consumer opinions with reservation prices in an agent-based model of innovation diffusion. Acta Phys. Pol. A 2016, 129, 1055-1059.

13. Maciejowska, K.; Jędrzejewski, A.; Kowalska-Pyzalska, A.; Weron, R. Impact of social interactions on demand curves for innovative products. Acta Phys. Pol. A 2016, 129, 1045-1049. 
14. Byrka, K.; Jędrzejewski, A.; Sznajd-Weron, K.; Weron, R. Difficulty is critical: The importance of social factors in modeling diffusion of green products and practices. Renew. Sust. Energy Rev. 2016, 62, 723-735.

15. Whalen, A.; Griffiths, T.L. Adding population structure to models of language evolution by iterated learning. J. Math. Psychol. 2017, 76, 1-6.

16. Clifford, P.; Sudbury, A. A model for spatial conflict. Biometrika 1973, 60, 581-588.

17. Myers, D.G. Social Psychology; The McGraw-Hill Companies, Inc.: New York, NY, USA, 2010.

18. Nail, P.R.; di Domenico, S.I.; MacDonald, G. Proposal of a double diamond model of social response. Rev. Gen. Psychol. 2013,17,1-19.

19. Lee, S.H.; Ha, M.; Jeong, H.; Noh, J.D.; Park, H. Critical behavior of the Ising model in annealed scale-free networks. Phys. Rev. E 2009, 80, 051127.

20. Ballesteros, F.J.; Luque, B. Order-disorder phase transition in random-walk networks. Phys. Rev. E 2005, 71, 031104.

21. Noël, P.A.; Brummitt, C.D.; D'Souza, R.M. Bottom-up model of self-organized criticality on networks. Phys. Rev. E 2014, 89, 012807.

22. Thorpe, M.; Beeman, D. Thermodynamics of an Ising model with random exchange interactions. Phys. Rev. B 1976, 14, 188.

23. Malmi-Kakkada, A.N.; Valls, O.T.; Dasgupta, C. Ising model on a random network with annealed or quenched disorder. Phys. Rev. B 2014, 90, 024202.

24. Bera, A.; Rakshit, D.; De, A.S.; Sen, U. Spontaneous magnetization of quantum XY spin model in joint presence of quenched and annealed disorder. Phys. Rev. B 2017, 95, 224441.

25. Caticha, N. From quenched to annealed: a study of the intermediate dynamics of disorder. J. Phys. A Math. Gen. 1994, 27, 5501.

26. Lipowski, A.; Gontarek, K.; Lipowska, D. Robust criticality of an Ising model on rewired directed networks. Phys. Rev. E 2015, 91, 062801.

27. Jakubczak, J.; Mrozinska, Z.; Pekalski, A. Critical temperature of mixed ferromagnetic and antiferromagnetic Ising systems with arbitrary spin. J. Phys. C Solid State Phys. 1979, 12, 2341.

28. Jędrzejewski, A.; Chmiel, A.; Sznajd-Weron, K. Kinetic Ising models with various single-spin flip dynamics on quenched and annealed random regular graphs. Phys. Rev. E 2017, 96, 012132.

29. Galam, S.; Moscovici, S. Towards a theory of collective phenomena: Consensus and attitude changes in groups. Eur. J. Soc. Psychol. 1991, 21, 49-74.

30. Galam, S. Rational group decision making: A random field Ising model at T=0. Physica A Stat. Mech. Appl. 1996, 238, 66-80.

31. Galam, S. Contrarian deterministic effects on opinion dynamics: the hung elections scenario. Physica A Stat. Mech. Appl. 2004, 333, 453-460.

32. Stauffer, D.; Sá Martins, J.S. Simulation of Galam's contrarian opinions on percolative lattices. Physica A Stat. Mech. Appl. 2004, 334, 558-565.

33. Borghesi, C.; Galam, S. Chaotic, staggered, and polarized dynamics in opinion forming: The contrarian effect. Phys. Rev. E 2006, 73, 066118.

34. Schneider, J. The influence of contrarians and opportunists on the stability of a democracy in the Sznajd model. Int. J. Mod. Phys. C 2004, 15, 659-674.

35. Javarone, M.A. Social influences in opinion dynamics: The role of conformity. Physica A Stat. Mech. Appl. 2014, 414, 19-30.

36. de la Lama, M.S.; Lopez, J.M.; Wio, H.S. Spontaneous emergence of contrarian-like behavior in an opinion spreading model. Europhys. Lett. 2005, 72, 851-857.

37. Galam, S.; Jacobs, F. The role of inflexible minorities in the breaking of democratic opinion dynamics. Physica A Stat. Mech. Appl. 2007, 381, 366-376.

38. Mobilia, M. Does a single zealot affect an infinite group of voters? Phys. Rev. Lett. 2003, 91, 028701.

39. Javarone, M.A.; Antonioni, A.; Caravelli, F. Conformity-driven agents support ordered phases in the spatial public goods game. EPL 2016, 114, 38001.

40. Goldstein, J.H. Social Psychology; Academic Press: Cambridge, MA, USA, 2013.

41. Fleeson, W. Toward a structure- and process-integrated view of personality: Traits as density distribution of states. J. Pers. Soc. Psychol. 2001, 80, 1011-1027. 
42. Javarone, M.A.; Squartini, T. Conformism-driven phases of opinion formation on heterogeneous networks: The q-voter model case. J. Stat. Mech. Theory Exp. 2015, 2015, P10002.

43. Nail, P.R.; Sznajd-Weron, K. The diamond model of social response within an agent-based approach. Acta Phys. Pol. A 2016, 129, 1050-1054.

44. Chatelain, C.; Berche, B. Finite-size scaling study of the surface and bulk critical behavior in the random-bond eight-state Potts model. Phys. Rev. Lett. 1998, 80, 1670-1673.

45. Ausloos, M.; Pekalski, A. Approximate equivalence of quenched and annealed random systems in the effective Hamiltonian approximation. J. Phys. A Math. Gen. 1980, 13, 2537-2540.

46. Thorpe, M.F.; Beerman, D. Thermodynamics of an Ising model with random exchange interactions. Phys. Rev. B 1976, 14, 188-199.

47. Brout, R. Statistical mechanical theory of a random ferromagnetic system. Phys. Rev. 1959, 115, 824-835.

48. Harris, A.B. Effect of random defects on the critical behaviour of Ising models. J. Phys. C 1974, 7, 1671-1692.

(c) 2017 by the authors. Licensee MDPI, Basel, Switzerland. This article is an open access article distributed under the terms and conditions of the Creative Commons Attribution (CC BY) license (http://creativecommons.org/licenses/by/4.0/). 